\title{
Darboux diagonalization of the spatial 3-metric in Kerr spacetime
}

\section{Joshua Baines ${ }^{\oplus}$, Thomas Berry ${ }^{\oplus}$, Alex Simpson ${ }^{\circledR}$, and Matt Visser ${ }^{\circledR}$}

School of Mathematics and Statistics, Victoria University of Wellington, PO Box 600, Wellington 6140, New Zealand.

E-mail: joshua.baines@sms.vuw.ac.nz, thomas.berry@sms.vuw.ac.nz, alex.simpson@sms.vuw.ac.nz, matt.visser@sms.vuw.ac.nz

ABstract:

The astrophysical importance of the Kerr spacetime cannot be overstated. Of the currently known exact solutions to the Einstein field equations, the Kerr spacetime stands out in terms of its direct applicability to describing astronomical black hole candidates. In counterpoint, purely mathematically, there is an old classical result of differential geometry, due to Darboux, that all 3-manifolds can have their metrics recast into diagonal form. In the case of the Kerr spacetime the Boyer-Lindquist coordinates provide an explicit example of a diagonal spatial 3-metric. Unfortunately, as we demonstrate herein, Darboux diagonalization of the spatial 3-slices of the Kerr spacetime is incompatible with simultaneously putting the Kerr metric into unitlapse form while retaining manifest axial symmetry. This no-go theorem is somewhat reminiscent of the no-go theorem to the effect that the spatial 3-slices of the Kerr spacetime cannot be chosen to be conformally flat.

DATE: Thursday 3 September 2020; HTEX-ed September 4, 2020

KEYWORDS:

Kerr spacetime; Painlevé-Gullstrand coordinates; Boyer-Lindquist coordinates; Doran coordinates; ADM decomposition; unit lapse; Darboux diagonalization; 3-metric.

PHYSH: Gravitation 


\section{Contents}

1 Introduction 1

2 Framework and Setup 2

3 Azimuth-only coordinate transformations 3

4 Polar-only $(r, \theta)$ coordinate transformations $\quad 4$

5 Axisymmetry preserving coordinate transformations 5

6 Discussion and Conclusions $\quad 8$

A Appendix: Some explicit metrics $\quad 9$

A.1 Unit-lapse metrics 9

A.2 Lense-Thirring (Painlevé-Gullstrand version) 9

$\begin{array}{lll}\text { A.3 Boyer-Lindquist } & 10\end{array}$

$\begin{array}{lll}\text { A.4 Boyer-Lindquist-rain } & 10\end{array}$

A.5 Eddington-Finklestein-rain 11

$\begin{array}{lll}\text { A.6 Doran } & 11\end{array}$

\section{Introduction}

The Kerr spacetime [1-5] is perhaps the single most astrophysically important and pre-eminent of the currently known exact solutions to the Einstein field equations. (For general background discussion see textbooks such as [6-13].) In view of this central physical importance of the Kerr spacetime, it is well worth developing as many distinct viewpoints [14], alternative presentations [15-19], and tractable approximations [20-22] to the Kerr spacetime as possible. We have recently considered various unit-lapse "rain" representations of Kerr [17], and now wish to address the interplay between unit-lapse and possible diagonalization of the spatial 3-metric.

It is a classic mathematical result, typically attributed to Darboux [23], that under mild conditions 3-manifolds can have their metrics recast in diagonal form [24-29]. The question we wish to raise is whether Darboux diagonalization of the spatial slices of the Kerr spacetime is compatible with simultaneously maintaining the unit lapse condition and axial symmetry. When starting the calculation there were both reasons for optimism and reasons for caution. On the one hand:

- Diagonalization of the 3-metric only imposes 3 coordinate conditions on the 4metric, while the unit lapse condition only adds 1 more. Imposing 4 coordinate conditions in $(3+1)$ spacetime naively seems plausible. 
- Boyer-Lindquist coordinates for Kerr explicitly diagonalize the 3-metric, though they are not unit-lapse coordinates.

- Many unit-lapse coordinates for Kerr are known to exist [15-17], including unit-lapse coordinates very closely related to Boyer-Lindquist coordinates [17].

- The Lense-Thirring approximation to Kerr can explicitly be cast in unit-lapse flat 3-space form [22].

On the other hand, the Kerr spacetime sometimes exhibits (perhaps unexpected) obstructions to otherwise plausible conjectures. For instance, all static spherically symmetric (and some stationary axisymmetric, e.g., Lense-Thirring) spacetimes can be put into Painlevé-Gullstrand [30-32] form, with a flat spatial 3-metric [22, 33]. But not Kerr. Indeed, the spatial 3-slices of Kerr cannot even be put in conformally flat form $[34,35]$.

Unfortunately in the situation that we are interested in, the Kerr spacetime again is problematic — we shall demonstrate that in the Kerr spacetime the unit-lapse condition is incompatible with diagonalizing the 3-metric while maintaining axial symmetry. This is more than just an effect of incompatible symmetries, (for instance, things do work nicely for Lense-Thirring spacetime), the incompatibility depends on specific properties of the metric coefficients, and so on specific dynamical features arising from solving the Einstein equations.

\section{Framework and Setup}

All 3 of the fully specific unit-lapse versions of Kerr that we explored in reference [17], (BL-rain, EF-rain, Doran) have spatial 3-metrics of the form:

$$
d s^{2}=g_{r r}(r, \theta) d r^{2}+g_{\theta \theta}(r, \theta) d \theta^{2}+g_{\phi \phi}(r, \theta) d \phi^{2}+2 g_{r \phi}(r, \theta) d r d \phi .
$$

That is, the pattern of non-zero elements in the spatial 3-metric is:

$$
g_{i j}=\left[\begin{array}{ccc}
* & 0 & * \\
0 & * & 0 \\
* & 0 & *
\end{array}\right]_{i j}
$$

We wish to diagonalize this 3-metric, while preserving unit lapse and manifest axial symmetry. To preserve unit lapse, do not touch the time coordinate [17]. To preserve axial symmetry, transformations of the $\phi$ coordinate are limited to

$$
\phi=\bar{\phi}+\Phi(\bar{r}, \bar{\theta}) ; \quad d \phi=d \bar{\phi}+\Phi_{\bar{r}} d \bar{r}+\Phi_{\bar{\theta}} d \bar{\theta} .
$$


In principle we could do arbitrary things to $r$ and $\theta$ :

$$
(r, \theta) \rightarrow(\bar{r}, \bar{\theta})
$$

But let us take the analysis one step at a time.

\section{Azimuth-only coordinate transformations}

Let us temporarily agree to leave the $r$ and $\theta$ coordinates untouched; only adjust $\phi$. That is, we consider:

$$
r=\bar{r} ; \quad \theta=\bar{\theta} ; \quad \phi=\bar{\phi}+\Phi(\bar{r}, \bar{\theta})
$$

Thence

$$
d r=d \bar{r} ; \quad d \theta=d \bar{\theta} ; \quad d \phi=d \bar{\phi}+\Phi_{\bar{r}} d \bar{r}+\Phi_{\bar{\theta}} d \bar{\theta} .
$$

So for the 3-metric

$$
\begin{aligned}
d s^{2} & =g_{r r}(\bar{r}, \bar{\theta}) d \bar{r}^{2}+g_{\theta \theta}(\bar{r}, \bar{\theta}) d \bar{\theta}^{2} \\
& +g_{\phi \phi}(\bar{r}, \bar{\theta})\left(d \bar{\phi}+\Phi_{\bar{r}} d \bar{r}+\Phi_{\bar{\theta}} d \bar{\theta}\right)^{2} \\
& +2 g_{r \phi}(\bar{r}, \bar{\theta}) d \bar{r}\left(d \bar{\phi}+\Phi_{\bar{r}} d \bar{r}+\Phi_{\bar{\theta}} d \bar{\theta}\right)
\end{aligned}
$$

Now just pick off the 3 off-diagonal components:

$$
\begin{aligned}
g_{\bar{r} \bar{\phi}} & =g_{r \phi}(\bar{r}, \bar{\theta})+g_{\phi \phi}(\bar{r}, \bar{\theta}) \Phi_{\bar{r}} \\
g_{\bar{\theta} \bar{\phi}} & =g_{\phi \phi}(\bar{r}, \bar{\theta}) \Phi_{\bar{\theta}} \\
g_{\bar{r} \bar{\theta}} & =\Phi_{\bar{\theta}}\left(g_{\phi \phi}(\bar{r}, \bar{\theta}) \Phi_{\bar{r}}+g_{r \phi}(\bar{r}, \bar{\theta})\right) .
\end{aligned}
$$

We want all 3 of $g_{\bar{r} \bar{\phi}}, g_{\bar{\theta} \bar{\phi}}$, and $g_{\bar{r} \bar{\theta}}$ to vanish. That is, we demand that the 3 PDEs below must be satisfied:

$$
\begin{array}{ll}
E 1: & g_{r \phi}(\bar{r}, \bar{\theta})+g_{\phi \phi}(\bar{r}, \bar{\theta}) \Phi_{\bar{r}}=0 ; \\
E 2: & g_{\phi \phi}(\bar{r}, \bar{\theta}) \Phi_{\bar{\theta}}=0 ; \\
E 3: & \Phi_{\bar{\theta}}\left(g_{\phi \phi}(\bar{r}, \bar{\theta}) \Phi_{\bar{r}}+g_{r \phi}(\bar{r}, \bar{\theta})\right)=0 .
\end{array}
$$

But, since $g_{\phi \phi} \neq 0, E 2$ implies $\Phi_{\bar{\theta}}=0$, which then automatically satisfies $E 3$.

But this also implies $\Phi(\bar{r}, \bar{\theta}) \rightarrow \Phi(\bar{r})$, and $E 1$ becomes:

$$
\frac{d \Phi(\bar{r})}{d \bar{r}}=-\frac{g_{r \phi}(\bar{r}, \bar{\theta})}{g_{\phi \phi}(\bar{r}, \bar{\theta})}
$$


This equation is consistent iff

$$
\partial_{\bar{\theta}}\left(\frac{g_{r \phi}(\bar{r}, \bar{\theta})}{g_{\phi \phi}(\bar{r}, \bar{\theta})}\right)=0 .
$$

But inspection of the BL-rain, EF-rain, or Doran unit-lapse version of Kerr shows that this consistency condition is not satisfied. (See the appendix for more details.) Specifically, (recalling that in this section $\bar{r}=r$ and $\bar{\theta}=\theta$ ), we would want to have

$$
\partial_{\theta}\left(\frac{g_{r \phi}(r, \theta)}{g_{\phi \phi}(r, \theta)}\right)=0
$$

However

$$
\begin{aligned}
& \left(\frac{g_{r \phi}(r, \theta)}{g_{\phi \phi}(r, \theta)}\right)_{\text {BL-rain }}=\frac{2 m a r \sqrt{2 m r\left(r^{2}+a^{2}\right)}}{\Delta} \frac{1}{\rho^{2} \Sigma} \\
& \left(\frac{g_{r \phi}(r, \theta)}{g_{\phi \phi}(r, \theta)}\right)_{\text {EF-rain }}=-\frac{a}{\Sigma}\left(\frac{1+2 m r / \rho^{2}+\sqrt{2 m r /\left(r^{2}+a^{2}\right)}}{1+\sqrt{2 m r /\left(r^{2}+a^{2}\right)}}\right) \\
& \left(\frac{g_{r \phi}(r, \theta)}{g_{\phi \phi}(r, \theta)}\right)_{\text {Doran }}=-\frac{a}{\Sigma} \sqrt{\frac{2 m r}{a^{2}+r^{2}}} .
\end{aligned}
$$

Here $\rho^{2}=r^{2}+a^{2} \cos ^{2} \theta$, and $\Delta=r^{2}+a^{2}-2 m r$, while $\Sigma=r^{2}+a^{2}+\frac{2 m r a^{2}}{\rho^{2}} \sin ^{2} \theta$. It is the explicit presence of $\theta$ inside $\Sigma$ that is the obstruction to satisfying the consistency condition. So no azimuth-only coordinate transformation is capable of diagonalizing the spatial 3-metric. (We have been rather slow and careful with this calculation to make the general pattern clear.)

\section{Polar-only $(r, \theta)$ coordinate transformations}

Now let us leave the azimuthal coordinate intact, and only transform the $(r, \theta)$ plane. That is, we consider:

$$
r=G(\bar{r}, \bar{\theta}) ; \quad \theta=H(\bar{r}, \bar{\theta}) ; \quad \phi=\bar{\phi}
$$

Thence

$$
d r=G_{\bar{r}} d \bar{r}+G_{\bar{\theta}} d \bar{\theta} ; \quad d \theta=H_{\bar{r}} d \bar{r}+H_{\bar{\theta}} d \bar{\theta} ; \quad d \phi=d \bar{\phi} .
$$

So for the 3-metric

$$
\begin{aligned}
d s^{2} & =g_{r r}(G(\bar{r}, \bar{\theta}), H(\bar{r}, \bar{\theta}))\left(G_{\bar{r}} d \bar{r}+G_{\bar{\theta}} d \bar{\theta}\right)^{2} \\
& +g_{\theta \theta}(G(\bar{r}, \bar{\theta}), H(\bar{r}, \bar{\theta}))\left(H_{\bar{r}} d \bar{r}+H_{\bar{\theta}} d \bar{\theta}\right)^{2} \\
& +g_{\phi \phi}(G(\bar{r}, \bar{\theta}), H(\bar{r}, \bar{\theta})) d \bar{\phi}^{2} \\
& +2 g_{r \phi}(G(\bar{r}, \bar{\theta}), H(\bar{r}, \bar{\theta}))\left(G_{\bar{r}} d \bar{r}+G_{\bar{\theta}} d \bar{\theta}\right) d \bar{\phi}
\end{aligned}
$$


Now to simplify the notation write $g_{i j}(G(\bar{r}, \bar{\theta}), H(\bar{r}, \bar{\theta})) \rightarrow g_{i j}(\bar{r}, \bar{\theta})$ and then just pick off the 3 off-diagonal components:

$$
\begin{aligned}
g_{\bar{r} \bar{\phi}} & =g_{r \phi}(\bar{r}, \bar{\theta}) G_{\bar{r}} \\
g_{\bar{\theta} \bar{\phi}} & =g_{r \phi}(\bar{r}, \bar{\theta}) G_{\bar{\theta}} \\
g_{\bar{r} \bar{\theta}} & =g_{r r}(\bar{r}, \bar{\theta}) G_{\bar{\theta}} G_{\bar{r}}+g_{\theta \theta}(\bar{r}, \bar{\theta}) H_{\bar{r}} H_{\bar{\theta}} .
\end{aligned}
$$

Now we want all 3 of $g_{\bar{r} \bar{\phi}}, g_{\bar{\theta} \bar{\phi}}$, and $g_{\bar{r} \bar{\theta}}$ to vanish.

That is we demand that the 3 PDEs below must be satisfied:

$$
\begin{array}{ll}
E 1: & g_{r \phi}(\bar{r}, \bar{\theta}) G_{\bar{r}}=0 \\
E 2: & g_{r \phi}(\bar{r}, \bar{\theta}) G_{\bar{\theta}}=0 ; \\
E 3: & g_{r r}(\bar{r}, \bar{\theta}) G_{\bar{\theta}} G_{\bar{r}}+g_{\theta \theta}(\bar{r}, \bar{\theta}) H_{\bar{r}} H_{\bar{\theta}}=0 .
\end{array}
$$

But, since we know $g_{r \phi} \neq 0$, then $E 1$ implies $G_{\bar{r}}=0$, and $E 2$ implies $G_{\bar{\theta}}=0$. But then $G(\bar{r}, \bar{\theta})$ is a constant; so it is not a good coordinate - we have an inconsistency. (We do not even need to look at equation E3.) So we cannot diagonalize the spatial 3 -metric using only polar $(r, \theta)$ coordinate transformations.

\section{Axisymmetry preserving coordinate transformations}

Now consider the general case - this is the most general thing one can do without damaging the manifest axial symmetry. Consider:

$$
r=G(\bar{r}, \bar{\theta}) ; \quad \theta=H(\bar{r}, \bar{\theta}) ; \quad \phi=\bar{\phi}+\Phi(\bar{r}, \bar{\theta}) .
$$

Thence

$$
d r=G_{\bar{r}} d \bar{r}+G_{\bar{\theta}} d \bar{\theta} ; \quad d \theta=H_{\bar{r}} d \bar{r}+H_{\bar{\theta}} d \bar{\theta} ; \quad d \phi=d \bar{\phi}+\Phi_{\bar{r}} d \bar{r}+\Phi_{\bar{\theta}} d \bar{\theta}
$$

So for the 3-metric

$$
\begin{aligned}
d s^{2} & =g_{r r}(G(\bar{r}, \bar{\theta}), H(\bar{r}, \bar{\theta}))\left(G_{\bar{r}} d \bar{r}+G_{\bar{\theta}} d \bar{\theta}\right)^{2} \\
& +g_{\theta \theta}(G(\bar{r}, \bar{\theta}), H(\bar{r}, \bar{\theta}))\left(H_{\bar{r}} d \bar{r}+H_{\bar{\theta}} d \bar{\theta}\right)^{2} \\
& +g_{\phi \phi}(G(\bar{r}, \bar{\theta}), H(\bar{r}, \bar{\theta}))\left(d \bar{\phi}+\Phi_{\bar{r}} d \bar{r}+\Phi_{\bar{\theta}} d \bar{\theta}\right)^{2} \\
& +2 g_{r \phi}(G(\bar{r}, \bar{\theta}), H(\bar{r}, \bar{\theta}))\left(G_{\bar{r}} d \bar{r}+G_{\bar{\theta}} d \bar{\theta}\right)\left(d \bar{\phi}+\Phi_{\bar{r}} d \bar{r}+\Phi_{\bar{\theta}} d \bar{\theta}\right)
\end{aligned}
$$


Now to simplify the notation write $g_{i j}(G(\bar{r}, \bar{\theta}), H(\bar{r}, \bar{\theta})) \rightarrow g_{i j}(\bar{r}, \bar{\theta})$. That is

$$
\begin{aligned}
d s^{2} & =g_{r r}(\bar{r}, \bar{\theta})\left(G_{\bar{r}} d \bar{r}+G_{\bar{\theta}} d \bar{\theta}\right)^{2} \\
& +g_{\theta \theta}(\bar{r}, \bar{\theta})\left(H_{\bar{r}} d \bar{r}+H_{\bar{\theta}} d \bar{\theta}\right)^{2} \\
& +g_{\phi \phi}(\bar{r}, \bar{\theta})\left(d \bar{\phi}+\Phi_{\bar{r}} d \bar{r}+\Phi_{\bar{\theta}} d \bar{\theta}\right)^{2} \\
& +2 g_{r \phi}(\bar{r}, \bar{\theta})\left(G_{\bar{r}} d \bar{r}+G_{\bar{\theta}} d \bar{\theta}\right)\left(d \bar{\phi}+\Phi_{\bar{r}} d \bar{r}+\Phi_{\bar{\theta}} d \bar{\theta}\right) .
\end{aligned}
$$

Now just pick off the 3 off-diagonal components:

$$
\begin{aligned}
g_{\bar{r} \bar{\phi}}= & g_{r \phi}(\bar{r}, \bar{\theta}) G_{\bar{r}}+g_{\phi \phi}(\bar{r}, \bar{\theta}) \Phi_{\bar{r}} ; \\
g_{\bar{\theta} \bar{\phi}}= & g_{r \phi}(\bar{r}, \bar{\theta}) G_{\bar{\theta}}+g_{\phi \phi}(\bar{r}, \bar{\theta}) \Phi_{\bar{\theta}} ; \\
g_{\bar{r} \bar{\theta}}= & g_{r r}(\bar{r}, \bar{\theta}) G_{\bar{\theta}} G_{\bar{r}}+g_{\theta \theta}(\bar{r}, \bar{\theta}) H_{\bar{r}} H_{\bar{\theta}}+g_{\phi \phi}(\bar{r}, \bar{\theta}) \Phi_{\bar{r}} \Phi_{\bar{\theta}} \\
& +g_{r \phi}(\bar{r}, \bar{\theta})\left[\Phi_{\bar{r}} G_{\bar{\theta}}+\Phi_{\bar{\theta}} G_{\bar{r}}\right] .
\end{aligned}
$$

We want all 3 of $g_{\bar{r} \bar{\phi}}, g_{\bar{\theta} \bar{\phi}}$, and $g_{\bar{r} \bar{\theta}}$ to vanish. That is we demand that the 3 PDEs below must be satisfied:

$$
\begin{array}{ll}
E 1: & g_{r \phi}(\bar{r}, \bar{\theta}) G_{\bar{r}}+g_{\phi \phi}(\bar{r}, \bar{\theta}) \Phi_{\bar{r}}=0 ; \\
E 2: & g_{r \phi}(\bar{r}, \bar{\theta}) G_{\bar{\theta}}+g_{\phi \phi}(\bar{r}, \bar{\theta}) \Phi_{\bar{\theta}}=0 ; \\
E 3: & g_{r r}(\bar{r}, \bar{\theta}) G_{\bar{\theta}} G_{\bar{r}}+g_{\theta \theta}(\bar{r}, \bar{\theta}) H_{\bar{r}} H_{\bar{\theta}}+g_{\phi \phi}(\bar{r}, \bar{\theta}) \Phi_{\bar{r}} \Phi_{\bar{\theta}} \\
& +g_{r \phi}(\bar{r}, \bar{\theta})\left[\Phi_{\bar{r}} G_{\bar{\theta}}+\Phi_{\bar{\theta}} G_{\bar{r}}\right]=0 .
\end{array}
$$

These are 3 PDEs for 3 unknown functions. This is not, by itself, necessarily problematic. However, consider the specific linear combination

$$
\frac{1}{g_{\phi \phi}(\bar{r}, \bar{\theta})}\left(G_{\bar{\theta}} E 1-G_{\bar{r}} E 2\right)
$$

Then

$$
G_{\bar{\theta}} \Phi_{\bar{r}}-G_{\bar{r}} \Phi_{\bar{\theta}}=0 .
$$

This means that the cross product vanishes:

$$
\left(\Phi_{\bar{r}}, \Phi_{\bar{\theta}}\right) \times\left(G_{\bar{r}}, G_{\bar{\theta}}\right)=0
$$

This implies

$$
\left(\Phi_{\bar{r}}, \Phi_{\bar{\theta}}\right) \propto\left(G_{\bar{r}}, G_{\bar{\theta}}\right)
$$

With general solution

$$
\Phi(\bar{r}, \bar{\theta})=W(G(\bar{r}, \bar{\theta})),
$$

for some arbitrary function $W(G)$. 
Now substitute this back into $E 1$ and $E 2$ :

$$
\begin{array}{ll}
E 1^{\prime}: & G_{\bar{r}}(\bar{r}, \bar{\theta})\left[g_{r \phi}(\bar{r}, \bar{\theta})+g_{\phi \phi}(\bar{r}, \bar{\theta}) W^{\prime}(G(\bar{r}, \bar{\theta}))\right]=0 . \\
E 2^{\prime}: & G_{\bar{\theta}}(\bar{r}, \bar{\theta})\left[g_{r \phi}(\bar{r}, \bar{\theta})+g_{\phi \phi}(\bar{r}, \bar{\theta}) W^{\prime}(G(\bar{r}, \bar{\theta}))\right]=0 .
\end{array}
$$

Now we cannot have both $G_{\bar{r}}=0$ and $G_{\bar{\theta}}=0$, since that would mean $G$ is a constant, and so not a good coordinate. Therefore we must have

$$
\left[g_{r \phi}(\bar{r}, \bar{\theta})+g_{\phi \phi}(\bar{r}, \bar{\theta}) W^{\prime}(G(\bar{r}, \bar{\theta}))\right]=0 .
$$

That is:

$$
W^{\prime}(G(\bar{r}, \bar{\theta}))=-\frac{g_{r \phi}(\bar{r}, \bar{\theta})}{g_{\phi \phi}(\bar{r}, \bar{\theta})} .
$$

But unwrapping this in terms of the original coordinate system we started with, this means we are demanding

$$
W^{\prime}(r)=-\frac{g_{r \phi}(r, \theta)}{g_{\phi \phi}(r, \theta)} .
$$

But inspection of the BL-rain, EF-rain, or Doran unit-lapse versions of Kerr shows that this consistency condition is not satisfied. (The right hand side is explicitly $\theta$ dependent.) We have

$$
\begin{aligned}
&\left(\frac{g_{r \phi}(r, \theta)}{g_{\phi \phi}(r, \theta)}\right)_{\text {BL-rain }}=\frac{2 m a r \sqrt{2 m r\left(r^{2}+a^{2}\right)}}{\Delta} \frac{1}{\rho^{2} \Sigma} ; \\
&\left(\frac{g_{r \phi}(r, \theta)}{g_{\phi \phi}(r, \theta)}\right)_{\text {EF-rain }}=-\frac{a}{\Sigma}\left(\frac{1+2 m r / \rho^{2}+\sqrt{2 m r /\left(r^{2}+a^{2}\right)}}{1+\sqrt{2 m r /\left(r^{2}+a^{2}\right)}}\right) ; \\
&\left(\frac{g_{r \phi}(r, \theta)}{g_{\phi \phi}(r, \theta)}\right)_{\text {Doran }}=-\frac{a}{\Sigma} \sqrt{\frac{2 m r}{a^{2}+r^{2}}} .
\end{aligned}
$$

The $\theta$ dependence is hiding in $\Sigma$. (See appendix for details on these three metrics. Note the very strong similarities to the azimuth-only argument in section 3 above.) So no axisymmetry-preserving coordinate transformation is capable of diagonalizing the spatial metric.

Note that knowing the explicit forms of $g_{r \phi}(r, \theta)$ and $g_{\phi \phi}(r, \theta)$ in any one of the three BL-rain, EF-rain, or Doran unit-lapse versions of Kerr is enough to get to this conclusion. Also note that we never had to use equation E3. This completes the argument - in the unit-lapse Kerr context the Darboux diagonalization argument is incompatible with manifest axisymmetry. 


\section{Discussion and Conclusions}

What have we learned from this discussion? On the one hand, this no-go theorem is a very specific mathematical result specifically for the Kerr spacetime. On the other hand, the various ingredients that go into the discussion have a very much wider realm of applicability. For instance, the unit-lapse spacetimes occur quite commonly and very naturally in many specific examples of analogue spacetimes [36-52]. In an analogue spacetime context the unit lapse condition physically corresponds to a constant signal propagation speed. (This holds, for example, to a good approximation for sound waves in water.) Various analogue spacetimes can then be invoked to help one develop physical intuition in the current more purely general relativistic context, directly relevant to modelling infall and accretion.

Astrophysically, unit lapse versions of stationary spacetimes, (even if they are not vacuum solutions of the Einstein equations), are extremely useful in at they immediately provide an enormous class of timelike geodesics, the "rain geodesics". These correspond to the zero angular momentum observers, ZAMOs, that are dropped from spatial infinity with zero initial velocity and zero angular momentum. These rain geodesics provide an explicit and quite tractable probe of the spacetime physics.

The underlying theme behind our attempt at imposing Darboux diagonalization was to continue the search for improved coordinate systems for the Kerr spacetime. Finding such improved coordinate systems (if possible) is strategically and tactically important for a better understanding of the technically quite challenging and astrophysically important Kerr spacetime; see particularly the discussion in reference [3]. See also, for instance, recent attempts at finding a "Gordon form" for the Kerr spacetime [18], and recent attempts at upgrading the "Newman-Janis trick" from an ansatz to an algorithm [19].

Finally, we should also emphasise that the discussion herein also impacts on and informs the discussion and investigation regarding the potential observational ability to distinguish exact Kerr black holes from various "black hole mimickers" that have been suggested in the literature - see for instance references [53, 54]. More recently one could consider references [55-60], and references [61-71]. 


\section{A Appendix: Some explicit metrics}

In this appendix we list some of the explicit metrics utilized in the text.

\section{A.1 Unit-lapse metrics}

The general form for a unit-lapse metric is [17]:

$$
d s^{2}=-d t^{2}+h_{i j}\left(d x^{i}-v^{i} d t\right)\left(d x^{j}-v^{j} d t\right)
$$

Then

$$
g_{a b}=\left[\begin{array}{c|c}
-1+\left(h^{i j} v_{i} v_{j}\right) & -v_{j} \\
\hline-v_{i} & h_{i j}
\end{array}\right]_{a b} ; \quad g^{a b}=\left[\begin{array}{c|c}
-1 & -v^{j} \\
\hline-v^{i} & h^{i j}-v^{i} v^{j}
\end{array}\right]^{a b} .
$$

Note $\operatorname{det}\left(g_{a b}\right)=-\operatorname{det}\left(h_{i j}\right)$ and $g^{t t}=-1$.

\section{A.2 Lense-Thirring (Painlevé-Gullstrand version)}

For the Painlevé-Gullstrand version of Lense-Thirring [22]:

$$
d s^{2}=-d t^{2}+\left(d r+\sqrt{\frac{2 m}{r}} d t\right)^{2}+r^{2}\left(d \theta^{2}+\sin ^{2} \theta\left(d \phi-\frac{2 J}{r^{3}} d t\right)^{2}\right)
$$

Then

$$
g_{a b}=\left[\begin{array}{c|ccc}
-1+\frac{2 m}{r}+\frac{4 J^{2} \sin ^{2} \theta}{r^{4}} & \sqrt{\frac{2 m}{r}} & 0 & -\frac{2 J \sin ^{2} \theta}{r} \\
\hline \sqrt{\frac{2 m}{r}} & 1 & 0 & 0 \\
0 & 0 & r^{2} & 0 \\
-\frac{2 J \sin ^{2} \theta}{r} & 0 & 0 & r^{2} \sin ^{2} \theta
\end{array}\right]_{a b} .
$$

Here $h_{i j}$ is not just diagonal, it is flat 3 -space.

$$
g^{a b}=\left[\begin{array}{c|ccc}
-1 & \sqrt{\frac{2 m}{r}} & 0 & -\frac{2 J}{r^{3}} \\
\hline \sqrt{\frac{2 m}{r}} & 1-\frac{2 m}{r} & 0 & \sqrt{\frac{2 m}{r}} \frac{2 J}{r^{3}} \\
0 & 0 & \frac{1}{r^{2}} & 0 \\
-\frac{2 J}{r^{3}} & \sqrt{\frac{2 m}{r}} \frac{2 J}{r^{3}} & 0 & \frac{1}{r^{2} \sin ^{2} \theta}-\frac{4 J^{2}}{r^{6}}
\end{array}\right]^{a b} .
$$

Note this is unit lapse, $g^{t t}=-1$. 


\section{A.3 Boyer-Lindquist}

The standard form for the Boyer-Lindquist version of Kerr is:

$$
\left(g_{a b}\right)_{B L}=\left[\begin{array}{c|cc|c}
-1+\frac{2 m r}{\rho^{2}} & 0 & 0 & -\frac{2 m a r \sin ^{2} \theta}{\rho^{2}} \\
\hline 0 & \frac{\rho^{2}}{\Delta} & 0 & 0 \\
0 & 0 & \rho^{2} & 0 \\
\hline-\frac{2 m a r \sin ^{2} \theta}{\rho^{2}} & 0 & 0 & \Sigma \sin ^{2} \theta
\end{array}\right]_{a b} .
$$

Note $h_{i j}$ is diagonal.

$$
\left(g^{a b}\right)_{B L}=\left[\begin{array}{c|cc|c}
-1-\frac{2 m r\left(r^{2}+a^{2}\right)}{\rho^{2} \Delta} & 0 & 0 & -\frac{2 m a r}{\rho^{2} \Delta} \\
\hline 0 & \frac{\Delta}{\rho^{2}} & 0 & 0 \\
0 & 0 & \frac{1}{\rho^{2}} & 0 \\
\hline-\frac{2 m a r}{\rho^{2} \Delta} & 0 & 0 & \frac{1-2 m r / \rho^{2}}{\Delta \sin ^{2} \theta}
\end{array}\right]^{a b} .
$$

Here $\rho^{2}=r^{2}+a^{2} \cos ^{2} \theta$, and $\Delta=r^{2}+a^{2}-2 m r$, while $\Sigma=r^{2}+a^{2}+\frac{2 m r a^{2}}{\rho^{2}} \sin ^{2} \theta$.

Note this is not unit lapse, $g^{t t} \neq-1$.

\section{A.4 Boyer-Lindquist-rain}

For the Boyer-Lindquist-rain version of the Kerr metric $\left(g_{a b}\right)_{\text {BL-rain }}$ we have [17]:

$$
\left[\begin{array}{c|cc|c}
-1+\frac{2 m r}{\rho^{2}} & \left(1-\frac{2 m r}{\rho^{2}}\right) \frac{\sqrt{2 m r\left(r^{2}+a^{2}\right)}}{\Delta} & 0 & -\frac{2 m a r \sin ^{2} \theta}{\rho^{2}} \\
\hline\left(1-\frac{2 m r}{\rho^{2}}\right) \frac{\sqrt{2 m r\left(r^{2}+a^{2}\right)}}{\Delta} & \frac{\rho^{2}}{\Delta}-\left(1-\frac{2 m r}{\rho^{2}}\right) \frac{2 m r\left(r^{2}+a^{2}\right)}{\Delta^{2}} & 0 & \frac{2 m a r \sin ^{2} \theta}{\rho^{2}} \frac{\sqrt{2 m r\left(r^{2}+a^{2}\right)}}{\Delta} \\
0 & 0 & \rho^{2} & 0 \\
\hline-\frac{2 m a r \sin ^{2} \theta}{\rho^{2}} & \frac{2 m a r \sin ^{2} \theta}{\rho^{2}} \frac{\sqrt{2 m r\left(r^{2}+a^{2}\right)}}{\Delta} & 0 & \sum \sin ^{2} \theta
\end{array}\right]_{a b}
$$

Note $h_{i j}$ is not diagonal.

For the inverse metric

$$
\left(g^{a b}\right)_{\text {BL-rain }}=\left[\begin{array}{c|cc|c}
-1 & \frac{\sqrt{2 m r\left(r^{2}+a^{2}\right)}}{\rho^{2}} & 0 & -\frac{2 m a r}{\rho^{2} \Delta} \\
\hline \frac{\sqrt{2 m r\left(r^{2}+a^{2}\right)}}{\rho^{2}} & \frac{\Delta}{\rho^{2}} & 0 & 0 \\
0 & 0 & \frac{1}{\rho^{2}} & 0 \\
\hline-\frac{2 m a r}{\rho^{2} \Delta} & 0 & 0 & \frac{1-2 m r / \rho^{2}}{\Delta \sin ^{2} \theta}
\end{array}\right]^{a b} .
$$

Note this is unit lapse, $g^{t t}=-1$. Oddly the spatial part of the inverse metric $g^{i j}$ is diagonal, but this is not the same as saying $h_{i j}$ is diagonal. 


\section{A.5 Eddington-Finklestein-rain}

In Eddington-Finkelstein-rain coordinates the covariant metric is given by [17]:

$$
\left(g_{a b}\right)_{\mathrm{EF-rain}}=\left[\begin{array}{c|cc|c}
-1+\frac{2 m r}{\rho^{2}} & g_{t r} & 0 & -\frac{2 m a r}{\rho^{2}} \sin ^{2} \theta \\
\hline g_{t r} & g_{r r} & 0 & g_{r \phi} \\
0 & 0 & \rho^{2} & 0 \\
\hline-\frac{2 m a r}{\rho^{2}} \sin ^{2} \theta & g_{r \phi} & 0 & \sum \sin ^{2} \theta
\end{array}\right]_{a b}
$$

subject to the relatively messy results that

$$
\begin{gathered}
g_{r r}=1+\frac{a^{2} \sin ^{2} \theta\left(2 m r / \rho^{2}\right)}{\left(r^{2}+a^{2}\right)\left(1+\sqrt{2 m r /\left(r^{2}+a^{2}\right)}\right)^{2}} ; \\
g_{t r}=\frac{2 m r / \rho^{2}+\sqrt{2 m r /\left(r^{2}+a^{2}\right)}}{1+\sqrt{2 m r /\left(r^{2}+a^{2}\right)}} ; \\
g_{r \phi}=-a \sin ^{2} \theta\left(\frac{1+2 m r / \rho^{2}+\sqrt{2 m r /\left(r^{2}+a^{2}\right)}}{1+\sqrt{2 m r /\left(r^{2}+a^{2}\right)}}\right) .
\end{gathered}
$$

Note $h_{i j}$ is not diagonal. The inverse metric is much simpler

$$
\left(g^{a b}\right)_{\text {EF-rain }}=\left[\begin{array}{c|cc|c}
-1 & \frac{\sqrt{2 m r\left(r^{2}+a^{2}\right)}}{\rho^{2}} & 0 & \frac{\sqrt{2 m r a^{2} /\left(r^{2}+a^{2}\right)}}{\rho^{2}\left(1+\sqrt{2 m r /\left(r^{2}+a^{2}\right)}\right)} \\
\hline \frac{\sqrt{2 m r\left(r^{2}+a^{2}\right)}}{\rho^{2}} & \frac{\Delta}{\rho^{2}} & 0 & \frac{a}{\rho^{2}} \\
0 & 0 & \frac{1}{\rho^{2}} & 0 \\
\hline \frac{\sqrt{2 m r a^{2} /\left(r^{2}+a^{2}\right)}}{\rho^{2}\left(1+\sqrt{2 m r /\left(r^{2}+a^{2}\right)}\right)} & \frac{a}{\rho^{2}} & 0 & \frac{1}{\rho^{2} \sin ^{2} \theta}
\end{array}\right]^{a b} .
$$

Note this is unit lapse, $g^{t t}=-1$.

\section{A.6 Doran}

The Doran metric is $[15,17]$

$$
\left(g_{a b}\right)_{\text {Doran }}=\left[\begin{array}{c|cc|c}
-1+\frac{2 m r}{\rho^{2}} & \sqrt{\frac{2 m r}{a^{2}+r^{2}}} & 0 & -\frac{2 m a r \sin ^{2} \theta}{\rho^{2}} \\
\hline \sqrt{\frac{2 m r}{a^{2}+r^{2}}} & \frac{\rho^{2}}{r^{2}+a^{2}} & 0 & -a \sqrt{\frac{2 m r}{a^{2}+r^{2}}} \sin ^{2} \theta \\
0 & 0 & \rho^{2} & 0 \\
\hline-\frac{2 m a r \sin ^{2} \theta}{\rho^{2}} & -a \sqrt{\frac{2 m r}{a^{2}+r^{2}}} \sin ^{2} \theta & 0 & \sum \sin ^{2} \theta
\end{array}\right]_{a b}
$$

Note $h_{i j}$ is not diagonal. 
For the inverse metric

$$
\left(g^{a b}\right)_{\text {Doran }}=\left[\begin{array}{c|cc|c}
-1 & \frac{\sqrt{2 m r\left(a^{2}+r^{2}\right)}}{\rho^{2}} & 0 & 0 \\
\hline \frac{\sqrt{2 m r\left(a^{2}+r^{2}\right)}}{\rho^{2}} & \frac{\Delta}{\rho^{2}} & 0 & \frac{a \sqrt{\frac{2 m r}{a^{2}+r^{2}}}}{\rho^{2}} \\
0 & 0 & \frac{1}{\rho^{2}} & 0 \\
\hline 0 & \frac{a \sqrt{\frac{2 m r}{a^{2}+r^{2}}}}{\rho^{2}} & 0 & \frac{1}{\left(a^{2}+r^{2}\right) \sin ^{2} \theta}
\end{array}\right]^{a b} .
$$

Note this is unit lapse, $g^{t t}=-1$.

\section{Acknowledgements}

JB was supported by a MSc scholarship funded by the Marsden Fund, via a grant administered by the Royal Society of New Zealand.

TB was supported by a Victoria University of Wellington MSc scholarship, and was also indirectly supported by the Marsden Fund, via a grant administered by the Royal Society of New Zealand.

AS was supported by a Victoria University of Wellington PhD Doctoral Scholarship, and was also indirectly supported by the Marsden fund, via a grant administered by the Royal Society of New Zealand.

MV was directly supported by the Marsden Fund, via a grant administered by the Royal Society of New Zealand.

\section{References}

[1] Roy Kerr, "Gravitational field of a spinning mass as an example of algebraically special metrics", Physical Review Letters 11 237-238 (1963).

[2] Roy Kerr, "Gravitational collapse and rotation", published in: Quasi-stellar sources and gravitational collapse: Including the proceedings of the First Texas Symposium on Relativistic Astrophysics, edited by Ivor Robinson, Alfred Schild, and E.L. Schücking (University of Chicago Press, Chicago, 1965), pages 99-102. The conference was held in Austin, Texas, on 16-18 December 1963.

[3] M. Visser, "The Kerr spacetime: A brief introduction", [arXiv:0706.0622 [gr-qc]]. Published in [4].

[4] D. L. Wiltshire, M. Visser and S. M. Scott, The Kerr spacetime: Rotating black holes in general relativity, (Cambridge University Press, Cambridge, 2009).

[5] Barrett O'Neill, The geometry of Kerr black holes, (Peters, Wellesley, 1995). Reprinted (Dover, Mineloa, 2014). 
[6] Ronald J. Adler, Maurice Bazin, and Menahem Schiffer, Introduction to General Relativity, Second edition, (McGraw-Hill, New York, 1975).

[It is important to acquire the 1975 second edition, the 1965 first edition does not contain any discussion of the Kerr spacetime.]

[7] Ray D'Inverno, Introducing Einstein's Relativity, (Oxford University Press, Oxford, 1992).

[8] James Hartle, Gravity: An introduction to Einstein's general relativity, (Addison Wesley, San Francisco, 2003).

[9] Sean Carroll, An introduction to general relativity: Spacetime and Geometry, (Addison Wesley, San Francisco, 2004).

[10] Robert Wald, General relativity, (University of Chicago Press, Chicago, 1984).

[11] Steven Weinberg, Gravitation and Cosmology: Principles and Applications of the General Theory of Relativity, (Wiley, Hoboken, 1972).

[12] M. P. Hobson, G. P. Estathiou, and A N. Lasenby, General relativity: An introduction for physicists, (Cambridge University Press, Cambridge, 2006).

[13] Charles Misner, Kip Thorne, and John Archibald Wheeler, Gravitation, (Freeman, San Francisco, 1973).

[14] A. J. Hamilton and J. P. Lisle, "The River model of black holes", Am. J. Phys. 76 (2008), 519-532 doi:10.1119/1.2830526 [arXiv:gr-qc/0411060 [gr-qc]].

[15] C. Doran, "A New form of the Kerr solution", Phys. Rev. D 61 (2000), 067503 doi:10.1103/PhysRevD.61.067503 [arXiv:gr-qc/9910099 [gr-qc]].

[16] J. Natario, "Painlevé-Gullstrand Coordinates for the Kerr Solution", Gen. Rel. Grav. 41 (2009), 2579-2586 doi:10.1007/s10714-009-0781-2 [arXiv:0805.0206 [gr-qc]].

[17] Joshua Baines, Thomas Berry, Alex Simpson, and Matt Visser, "Unit-lapse forms of the Kerr spacetime", arXiv:2008.03817 [gr-qc].

[18] S. Liberati, G. Tricella and M. Visser, "Towards a Gordon form of the Kerr spacetime", Class. Quant. Grav. 35 (2018) no.15, 155004 doi:10.1088/1361-6382/aacb75 [arXiv:1803.03933 [gr-qc]].

[19] D. Rajan and M. Visser, "Cartesian Kerr-Schild variation on the Newman-Janis trick", Int. J. Mod. Phys. D 26 (2017) no.14, 1750167 doi:10.1142/S021827181750167X [arXiv:1601.03532 [gr-qc]]. 
[20] Hans Thirring and Josef Lense,

"Über den Einfluss der Eigenrotation der Zentralkörperauf die Bewegung der

Planeten und Monde nach der Einsteinschen Gravitationstheorie",

Physikalische Zeitschrift, Leipzig Jg. 19 (1918), No. 8, p. 156-163.

English translation by Bahram Mashoon, Friedrich W. Hehl, and Dietmar S. Theiss, "On the influence of the proper rotations of central bodies on the motions of planets and moons in Einstein's theory of gravity",

General Relativity and Gravitation 16 (1984) 727-741.

[21] Herbert Pfister, "On the history of the so-called Lense-Thirring effect", http://philsci-archive.pitt.edu/archive/00002681/01/lense.pdf

[22] Joshua Baines, Thomas Berry, Alex Simpson, and Matt Visser, "Painlevé-Gullstrand form of the Lense-Thirring spacetime", arXiv:2006.14258 [gr-qc].

[23] Gaston Darboux,

"Leçons sur les systèmes orthogonaux et les coordonnées curvilignes", Gauthier-Villars, 1898.

[24] Luther Pfahler Eisenhart, "Fields of parallel vectors in a Riemannian geometry", Trans. Amer. Math. Soc. 27 (1925), no. 4, 563-573.

[25] D. M. DeTurck and D. Yang, "Existence of elastic deformations with prescribed principal strains and triply orthogonal systems",

Duke Math. J. 51 (1984), no. 2, 243-260.

[26] R. L. Bryant, S. S. Chern, R. B. Gardner, H. L. Goldschmidt and P. A. Griths, "Exterior differential systems", Mathematical Sciences Research Institute Publications, 18. (Springer-Verlag, New York, 1991).

[27] K. P. Tod, "On choosing coordinates to diagonalize the metric", Classical Quantum Gravity 9 (1992), no. 7, 1693-1705.

[28] Oldřich Kowalski and Masami Sekizawa, "Diagonalization of three-dimensional pseudo-Riemannian metrics", Journal of Geometry and Physics, 74 (December 2013) 251-255. doi: 10.1016/j.geomphys.2013.08.010

[29] J. D. E. Grant and J. A. Vickers, "Block diagonalization of four-dimensional metrics," Class. Quant. Grav. 26 (2009), 235014 doi:10.1088/0264-9381/26/23/235014 [arXiv:0809.3327 [math.DG]].

[30] Paul Painlevé, "La mécanique classique et la théorie de la relativité", C. R. Acad. Sci. (Paris) 173, 677?680(1921).

[31] Paul Painlevé, "La gravitation dans la mécanique de Newton et dans la mécanique d'Einstein", C.R Acad. Sci. (Paris) 173, 873-886(1921). 
[32] Gullstrand, Allvar (1922). "Allgemeine Lösung des statischen Einkörperproblems in der Einsteinschen Gravitationstheorie".

Arkiv för Matematik, Astronomi och Fysik. 16 (8): 1-15.

[33] K. Martel and E. Poisson,

"Regular coordinate systems for Schwarzschild and other spherical space-times",

Am. J. Phys. 69 (2001), 476-480 doi:10.1119/1.1336836

[arXiv:gr-qc/0001069 [gr-qc]].

[34] J. A. Valiente Kroon, "On the nonexistence of conformally flat slices in the Kerr and other stationary space-times," Phys. Rev. Lett. 92 (2004), 041101 doi:10.1103/PhysRevLett.92.041101 [arXiv:gr-qc/0310048 [gr-qc]].

[35] J. A. Valiente Kroon, "Asymptotic expansions of the Cotton-York tensor on slices of stationary space-times," Class. Quant. Grav. 21 (2004), 3237-3250 doi:10.1088/0264-9381/21/13/009 [arXiv:gr-qc/0402033 [gr-qc]].

[36] M. Visser, "Acoustic propagation in fluids: An unexpected example of Lorentzian geometry", [arXiv:gr-qc/9311028 [gr-qc]].

[37] M. Visser, "Acoustic black holes: Horizons, ergospheres, and Hawking radiation", Class. Quant. Grav. 15 (1998), 1767-1791 doi:10.1088/0264-9381/15/6/024 [arXiv:gr-qc/9712010 [gr-qc]].

[38] M. Visser, "Acoustic black holes", [arXiv:gr-qc/9901047 [gr-qc]].

[39] G. Volovik, "Simulation of Painlevé-Gullstrand black hole in thin He-3-A film", JETP Lett. 69 (1999), 705-713 doi:10.1134/1.568079 [arXiv:gr-qc/9901077 [gr-qc]].

[40] S. E. Perez-Bergliaffa, K. Hibberd, M. Stone and M. Visser, "Wave equation for sound in fluids with vorticity", Physica D 191 (2004), 121-136 doi:10.1016/j.physd.2003.11.007 [arXiv:cond-mat/0106255 [cond-mat]].

[41] M. Visser, C. Barceló and S. Liberati, "Analog models of and for gravity", Gen. Rel. Grav. 34 (2002), 1719-1734 doi:10.1023/A:1020180409214 [arXiv:gr-qc/0111111 [gr-qc]].

[42] U. R. Fischer and M. Visser, "On the space-time curvature experienced by quasiparticle excitations in the Painlevé-Gullstrand effective geometry", Annals Phys. 304 (2003), 22-39 doi:10.1016/S0003-4916(03)00011-3 [arXiv:cond-mat/0205139 [cond-mat]].

[43] M. Novello, M. Visser and G. Volovik, Artificial black holes, (World Scientific, Singapore, 2002)

[44] C. Barceló, S. Liberati and M. Visser, "Probing semiclassical analog gravity in Bose-Einstein condensates with widely tune-able interactions", Phys. Rev. A 68 (2003), 053613 doi:10.1103/PhysRevA.68.053613 [arXiv:cond-mat/0307491 [cond-mat]]. 
[45] M. Visser and S. E. C. Weinfurtner,

"Vortex geometry for the equatorial slice of the Kerr black hole",

Class. Quant. Grav. 22 (2005), 2493-2510 doi:10.1088/0264-9381/22/12/011

[arXiv:gr-qc/0409014 [gr-qc]].

[46] C. Barceló, S. Liberati and M. Visser, "Analogue gravity",

Living Rev. Rel. 8 (2005), 12 doi:10.12942/lrr-2005-12 [arXiv:gr-qc/0505065 [gr-qc]].

[47] S. Liberati, M. Visser and S. Weinfurtner,

"Analogue quantum gravity phenomenology from a two-component Bose-Einstein condensate", Class. Quant. Grav. 23 (2006), 3129-3154

doi:10.1088/0264-9381/23/9/023 [arXiv:gr-qc/0510125 [gr-qc]].

[48] S. Weinfurtner, S. Liberati and M. Visser,

"Analogue model for quantum gravity phenomenology",

J. Phys. A 39 (2006), 6807-6814 doi:10.1088/0305-4470/39/21/S83

[arXiv:gr-qc/0511105 [gr-qc]].

[49] M. Visser and C. Molina-París, "Acoustic geometry for general relativistic barotropic irrotational fluid flow", New J. Phys. 12 (2010), 095014 doi:10.1088/1367-2630/12/9/095014 [arXiv:1001.1310 [gr-qc]].

[50] M. Visser, "Survey of analogue spacetimes", Lect. Notes Phys. 870 (2013), 31-50 doi:10.1007/978-3-319-00266-8_2 [arXiv:1206.2397 [gr-qc]].

[51] S. Liberati, S. Schuster, G. Tricella and M. Visser, "Vorticity in analogue spacetimes",

Phys. Rev. D 99 \# 4 (2019) 044025 doi:10.1103/PhysRevD.99.044025

[arXiv:1802.04785 [gr-qc]].

[52] S. Schuster and M. Visser, "Boyer-Lindquist space-times and beyond:

Meta-material analogues", [arXiv:1802.09807 [gr-qc]].

[53] M. Visser, C. Barceló, S. Liberati and S. Sonego,

"Small, dark, and heavy: But is it a black hole?", PoS BHGRS (2008) 010

doi:10.22323/1.075.0010 [arXiv:0902.0346 [gr-qc]].

[54] M. Visser, "Black holes in general relativity", PoS BHGRS (2008), 001 doi:10.22323/1.075.0001 [arXiv:0901.4365 [gr-qc]].

[55] R. Carballo-Rubio, F. Di Filippo, S. Liberati and M. Visser, "Phenomenological aspects of black holes beyond general relativity", Phys. Rev. D 98 (2018) 124009. [arXiv:1809.08238 [gr-qc]].

[56] R. Carballo-Rubio, F. Di Filippo, S. Liberati, C. Pacilio and M. Visser, "On the viability of regular black holes", J. High Energ. Phys. 2018 (2018). [arXiv:1805.02675 [gr-qc]].

[57] R. Carballo-Rubio, F. Di Filippo, S. Liberati and M. Visser, "Geodesically complete black holes", Phys. Rev. D 101 (2020), 084047 doi:10.1103/PhysRevD.101.084047 [arXiv:1911.11200 [gr-qc]].

[58] R. Carballo-Rubio, F. Di Filippo, S. Liberati and M. Visser, "Opening the Pandora's box at the core of black holes", Class. Quant. Grav. 37 (2020) no.14, 145005 doi:10.1088/1361-6382/ab8141 [arXiv:1908.03261 [gr-qc]]. 
[59] R. Carballo-Rubio, F. Di Filippo, S. Liberati and M. Visser, "Causal hierarchy in modified gravity", [arXiv:2005.08533 [gr-qc]].

[60] E. Barausse, E. Berti, T. Hertog, S. A. Hughes, P. Jetzer, P. Pani, T. P. Sotiriou, N. Tamanini, H. Witek, K. Yagi, N. Yunes, et al., "Prospects for Fundamental Physics with LISA", doi:10.1007/s10714-020-02691-1 (GRG in press). [arXiv:2001.09793 [gr-qc]].

[61] F. S. N. Lobo, A. Simpson and M. Visser, "Dynamic thin-shell black-bounce traversable wormholes", Phys. Rev. D 101 (2020) no.12, 124035 doi:10.1103/PhysRevD.101.124035 [arXiv:2003.09419 [gr-qc]].

[62] A. Simpson, P. Martín-Moruno and M. Visser, "Vaidya spacetimes, black-bounces, and traversable wormholes", Class. Quant. Grav. 36 (2019) no.14, 145007 doi:10.1088/1361-6382/ab28a5 [arXiv:1902.04232 [gr-qc]].

[63] A. Simpson and M. Visser, "Black-bounce to traversable wormhole", JCAP 02 (2019), 042 doi:10.1088/1475-7516/2019/02/042 [arXiv:1812.07114 [gr-qc]].

[64] P. Boonserm, T. Ngampitipan, A. Simpson and M. Visser, "Exponential metric represents a traversable wormhole", Phys. Rev. D 98 (2018) no.8, 084048 doi:10.1103/PhysRevD.98.084048 [arXiv:1805.03781 [gr-qc]].

[65] A. Simpson and M. Visser, "Regular black holes with asymptotically Minkowski cores", Universe 6 (2019) no.1, 8 doi:10.3390/universe6010008 [arXiv:1911.01020 [gr-qc]].

[66] T. Berry, F. S. N. Lobo, A. Simpson and M. Visser, "Thin-shell traversable wormhole crafted from a regular black hole with asymptotically Minkowski core," [arXiv:2008.07046 [gr-qc]].

[67] T. Berry, A. Simpson and M. Visser, "Photon spheres, ISCOs, and OSCOs: Astrophysical observables for regular black holes with asymptotically Minkowski cores," [arXiv:2008.13308 [gr-qc]].

[68] J. M. Bardeen, "Non-singular general-relativistic gravitational collapse", in Proceedings of International Conference GR5, 1968, Tbilisi, USSR, p. 174.

[69] S. A. Hayward, "Formation and evaporation of regular black holes", Phys. Rev. Lett. 96 (2006) 031103 doi:10.1103/PhysRevLett.96.031103 [gr-qc/0506126].

[70] V. P. Frolov, "Information loss problem and a black hole model with a closed apparent horizon", JHEP 1405, 049 (2014) doi:10.1007/JHEP05(2014)049 [arXiv:1402.5446 [hep-th]].

[71] V. P. Frolov and A. Zelnikov, "Quantum radiation from an evaporating nonsingular black hole", Phys. Rev. D 95 (2017) no.12, 124028 doi:10.1103/PhysRevD.95.124028 [arXiv:1704.03043 [hep-th]]. 